\title{
Mixing Codebooks of LBG, KPE and KFCG Algorithms to Increase Capacity of Information Hiding
}

\author{
Dr. H. B. Kekre \\ Senior Professor, \\ MPSTME, NMIMS \\ University, Vile- \\ parle(W), Mumbai-56, \\ India.
}

\author{
Archana Athawale \\ Ph.D. Scholar MPSTME, \\ NMIMS University, \\ Mumbai-56, India. \\ Assistant Professor, \\ Thadomal Shahani \\ Engineering College, \\ Bandra(W), Mumbai-50, \\ India.
}

\author{
Dr. Tanuja K. Sarode \\ Ph.D. from MPSTME, \\ NMIMS University, \\ Mumbai-56, India. \\ Assistant Professor, \\ Thadomal Shahani \\ Engineering College, \\ Bandra(W), Mumbai-5, \\ India.
}

Kalpana Sagvekar

\author{
Lecturer, Fr. \\ Conceicao Rodrigues \\ COE, Bandra $(\mathrm{W})$, \\ Mumbai-50, India
}

\begin{abstract}
Many researchers have studied reversible data hiding techniques in recent years and most have proposed reversible data hiding schemes that guarantees only that the original cover image can be reconstructed completely. Once the secret data are embedded in the compression domain and the receiver wants to store the cover image in a compression mode to save storage space, the receiver must extract the secret data, reconstruct the cover image, and compress the cover image again to generate compression codes. In this paper, we propose a novel data hiding method based on VQ compressed images. Codebooks of secret message \& cover images are combined using shuffle algorithm. Experimental results indicate that our proposed scheme provides $100 \%$ hiding capacity or more that means secret message can be of same or more size than cover image and better image quality compared with existing schemes based on VQ compressed images. The technique is robust against stegaanalysis technique.
\end{abstract}

\section{General Terms}

Image Processing, Security

\section{Keywords}

Reversible Data hiding, VQ, LBG, KPE, KFCG.

\section{INTRODUCTION}

As the popularity of the Internet and the bandwidth increase rapidly, various digital multimedia can be transmitted over the Internet increasing day by day. Therefore, they can be copied easily through access to computer network and how to protect the digital multimedia security becomes more important every day. As usual, there are two widely used methods, one is the data encryption and the other is data hiding. In data encryption, the only particular user with the private key can recover the encryption message. Even though the attacker got an encryption message, it is also unable to find the content of message. Unfortunately, this method will be insecure when the private key is stolen or broken. Another way to solve this problem is to hide secret data behind a meaningful image such that an unintended observer will not be aware of the existence of the hidden secret message, i.e., it hides the secret data into a meaningful host data to distract the attention of the observers.

The existing schemes of data hiding can roughly be classified into the following three categories:

Spatial domain data hiding [2-4]: Data hiding of this type directly modifies image pixels in the spatial domain for data embedding. This technique is easy to implement, offers a relatively high hiding capacity, and the quality of the stego image can be easily controlled. Therefore, data hiding of this type has become a popular method for image steganography.

Frequency domain data hiding [5,6]: Images are first transformed into frequency domain, and then data are embedded by modifying the transformed coefficients. Frequency domain steganography often suffers from relatively higher computational cost and lower embedding capacity than those of spatial domain data hiding.

Compressed domain data hiding [7, 8]: Data hiding is achieved by modifying the coefficients of the compressed code of a cover image. Since most images transmitted over Internet are in compressed format, embedding secret data into the compressed domain would arouse little suspicion.

Information hiding techniques in spatial domain/transformed domain embed secrets by modifying spatial characteristics (i.e. pixel amplitude)/transformed coefficients of cover media. These techniques, however, are not robust enough to lossy compressions. Information hiding techniques in compressed domain (such as using vector quantization, VQ, compression method [10]) can make the transmitted data smaller [7] although there is a bit less data quantity to be embedded. In some sense, information hiding schemes can be further categorized into two types, namely irreversible data embedding schemes and reversible (or lossless) data embedding schemes. With respect to an irreversible data embedding scheme, receivers can extract secret data only, no cover media restoration. In contrast, with a reversible data embedding scheme, receivers can extract secret data and recover cover media completely [8]. A reversible hiding 
scheme is suitably used for the healthcare industry and online content distribution systems.

The rest of this paper is organized as follows: VQ compression techniques are briefly described in Section 2. Section 3 describes existing approach. The proposed data hiding is introduced in Section 4. Section 5 presents the experimental results of the proposed scheme. Finally, the conclusions are given in Section 6.

\section{VQ COMPRESSION TECHNIQUE}

Vector Quantization (VQ) [9-14] is an efficient technique for data compression [31-34] and is very popular in a variety of research fields such as image segmentation[23-26], speech data compression [27], content based image retrieval CBIR [28, 29] and face recognition [30].

\subsection{Codebook Generation Algorithms}

\subsubsection{Linde-Buzo-Gray (LBG) Algorithm [9], [10]}

In this algorithm centroid is computed as the first codevector for the training set. In Fig. 1 two vectors v1 \& v2 are generated by adding constant error to the codevector. Euclidean distances of all the training vectors are computed with vectors v1 \& v2 and two clusters are formed based on nearest of $\mathrm{v} 1$ or $\mathrm{v} 2$. This procedure is repeated for every cluster. The drawback of this algorithm is that the cluster elongation is $+135^{\circ}$ to horizontal axis in two dimensional cases. This results in inefficient clustering.

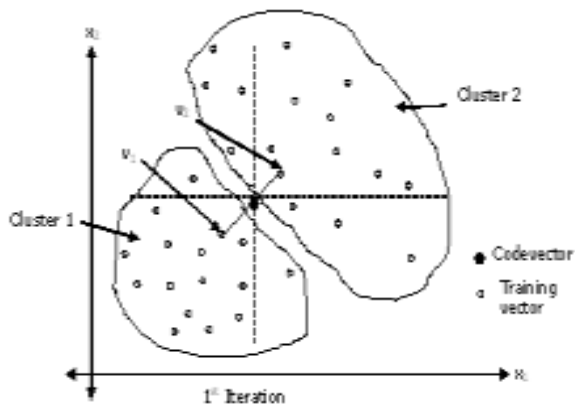

Figure 1: LBG for 2 dimensional case

\subsubsection{Proportionate Error Algorithm (KPE) [11], [12]}

Here proportionate error is added to the centroid to generate two vectors v1 \& v2. Magnitude of members of the centroid decides the error ratio. Hereafter the procedure is same as that of LBG. While adding proportionate error a safe guard is also introduced so that neither $\mathrm{v} 1$ nor v2 go beyond the training vector space. This removes the disadvantage of the LBG. Both LBG and KPE requires $2 \mathrm{M}$ number of Euclidean distance computations and $2 \mathrm{M}$ number of comparisons where $\mathrm{M}$ is the total number of training vectors in every iteration to generate clusters.

\subsubsection{Kekre's Fast Codebook Generation (KFCG) Algorithm [14]}

In [14], KFCG algorithm for image data compression is proposed. This algorithm reduces the time for codebook generation. It does not use Euclidian distance for codebook generation. In this algorithm image is divided in to blocks and blocks are converted to the vectors of size $\mathrm{k}$. Initially we have one cluster with the entire training vectors and the codevector $\mathrm{C} 1$ which is centroid.

In the first iteration of the algorithm, the clusters are formed by comparing first element of training vector with first element of code vector $C 1$. The vector $X_{i}$ is grouped into the cluster 1 if $x_{i 1}<$ $c_{11}$ otherwise vector $X_{i}$ is grouped into cluster 2 as shown in Figure 2(a). where codevector dimension space is 2 .

In second iteration, the cluster 1 is split into two by comparing second element $\mathrm{x}_{\mathrm{i} 2}$ of vector $\mathrm{X}_{\mathrm{i}}$ belonging to cluster 1 with that of the second element of the codevector which is centroid of cluster 1. Cluster 2 is split into two by comparing the second element $\mathrm{x}_{\mathrm{i} 2}$ of vector $\mathrm{X}_{\mathrm{i}}$ belonging to cluster 2 with that of the second element of the codevector which is centroid of cluster 2 , as shown in Figure 2(b).

This procedure is repeated till the codebook size is reached to the size specified by user. It is observed that this algorithm gives less error as compared to LBG and requires least time to generate codebook as compared to other algorithms, as it does not require computation of Euclidian distance.

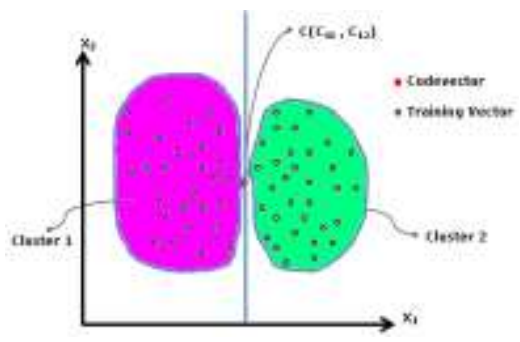

2(a) First Iteration

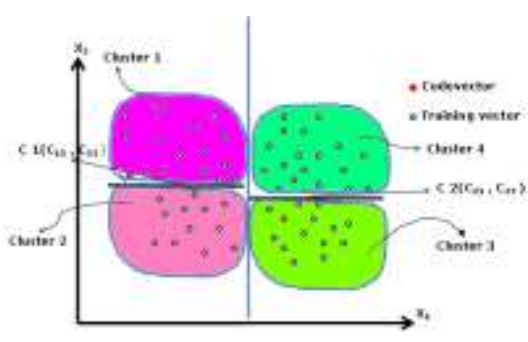

2(b) Second Iteration

Figure 2: KFCG algorithm for 2-D case

\section{EXISTING APPROACH - A BEST-PAIR- FIRST CAPACITY DISTORTION CONTROL FOR DATA HIDING ON VQ COMPRESSION DOMAIN [16]}

In Jo et al.'s [15] approach, each pair of code-vectors in $C B$ with the shortest Euclidean distance will be split into either groups $\left\{G_{0}\right.$, $\left.G_{1}\right\}$ or $G_{-1}$. In [16] approach, will perform the same splitting task. 
The difference is that group $G_{-1}$ will contain zero codevector by setting the threshold $t$ to a maximum value. Thus, each codevector $X_{i}$ in $G_{0}$ will always has a matching code-vector $X j$ in $G_{1}$ with the closest Euclidean distance. $X_{i}$ and $X_{j}$ is then treated as a matching pair of code-vectors. The proposed approach will then sort all matching pair of code-vectors based on their Euclidean distance in ascending order. A pair of code-vectors (also a pair of indices) is then assigned a label $w$ according to its sorting order. Thus, an index $i$ and its matching index $j$ both will have a smaller label $w$ as long as they have a smaller Euclidean distance. Since the codebook $C B$ contains $n$ code-vectors, the values of label $w$ will range from 1 to $n / 2$. Label $w$ will be used as the selection criterion for data embedding. That is, pair of VQ indices with smaller $w$ will be used to embed data first.

Based on the above preprocessing, the proposed best-pair-first approach for VQ compressing and data hiding can be described as follows.

\subsection{VQ encoding and data hiding}

Step 1: Perform VQ compression on image $I$ using codebook $C B$ as described in Section 2. This will generate a compressed file $F$ that contains a sequence of indices to serve as the VQ compression codes before data hiding.

Step 2: Let label $w=1$.

Step 3: Sequentially examine all indices in $F$ to find next index $I$ that was assigned a label $w$ during the preprocessing stage.

Step 4: Compare index $i$ with next secret bit $b$ in $S$. If $b=0$ and $i$ $\varepsilon G 0$, or $b=1$ and $i \varepsilon G 1$, leave index $i$ intact. Otherwise, change index $i$ in $F$ to be its matching index $j$ (for Alt $(X i)$ $=X j$ ).

Step 5: If there are more secret bits in $S$ to be embedded, repeat Step3 to Step4, until all indices in $F$ have been examined.

Step 6: If there are still more secret bits in $S$ to be embedded and $w<n / 2$, let $w=w+1$ and go to Step3. The result is a new compression file $F^{\prime}$ with the embedded message $S$

The idea is that indices in $F$ with smaller label $w$ will be used for embedding data, first. The embedding is done through alternating an index $i$ with its matching index $j$ based on the value of secret bit $b$ and the group $G_{0}$ or $G_{1}$ that index $i$ belongs to. The scanning and alternating of indices in $F$ will continue until all secret data in $S$ had been embedded.

\subsection{VQ decoding and data extraction}

The VQ decoding and data extracting for the proposed approach can be described as follows.

Step 1: For each index $i$ in $F^{\prime}$, perform a simple table look-up operation on the same codebook $C B$ to find its codevector $X_{i} . X_{i}$ is then served as the decoded image-vector for index $i$. The table look-up operation will continue until all image vectors have been recovered. The result of Step1 is the decoded stego-image $I^{\prime}$.
Step 2: $\quad$ Let label $w=1$.

Step 3: Sequentially examine all indices in $F^{\prime}$ to find next index $i$ that was assigned a label $w$ during the preprocessing stage.

Step 4: If $i \varepsilon G 0$, output a secret bit 0 , else output a secret bit 1 .

Step 5: If there are more secret bits in $S$ to be extracted, repeat Step3 to Step4, until all indices in $F^{\prime}$ had been examined.

Step 6: If there are still more secret bits in $S$ to be extracted and $w<n / 2$, let $w=w+1$ and go to Step3. As stated above, the stego-image $I^{\prime}$ can be decoded through the simple table look-up operation in Step1. Step2 to Step6 will extract secret message $S$ with the similar logic as in data embedding process.

\section{PROPOSED APPROACH}

Codebook of size N/2 is generated for cover image as well as secret image using codebook generation algorithm. Now two codebooks are merged to get mixed codebook of size $\mathrm{N}$ using shuffle algorithm which generates unique random numbers starting from 0 to $\mathrm{N}-1$.

Shuffle algorithm is as follows:

a) Select a distance $\mathrm{d}$ which is relatively prime to $\mathrm{N}$.

b) Start generating the random number starting from 0 . Numbers generated are $0, \mathrm{~d}, 2 \mathrm{~d}, 3 \mathrm{~d}$ and so on.

c) If number generated is $>\mathrm{N}$ then subtract $\mathrm{N}$ from it and take mod $d$ value as next random value. Go on adding $d$ to previous value to get the next value.

d) First N/2 random numbers are used for cover image and remaining $\mathrm{N} / 2$ for secret image.

If $\mathrm{N}$ is power of 2 then all odd numbers are relatively prime to $\mathrm{N}$. The mixed codebook \& distance $\mathrm{d}$ is sent to the receiver in order to reconstruct cover image $\&$ secret image by separating mixed codebook into individual codebook by generating unique random number upto $\mathrm{N}$ using distance $\mathrm{d}$.

\section{RESULTS \& EVALUATION}

In the existing approach, each input image-vector could hide one secret bit of data as described in Section 3. So, the VQ-hiding's upper bound is 16384 (i.e., $512 \times 512 / 16$ ) bits for a $512 \times 512$ gray-level cover image with the codebook CB of size $512 \times 16$. Total hiding capacity is only $6.25 \%$.

In our proposed approach, codebooks of cover image \& secret image are combined to get mixed codebook. So that hiding capacity is $100 \%$ or more since secret message can of same or larger size than cover image.

Figure 3 . shows the Cover images of size $256 \times 256$. In each cover image one secrete image given in Figure 4 is hidden.

Figure 4 shows the Secrete images of size $256 \times 256$ 


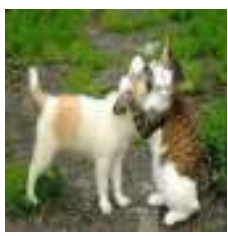

(a)

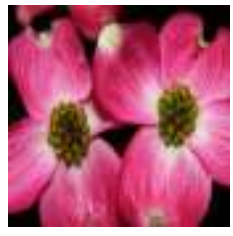

(c)

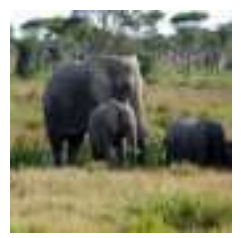

(b)

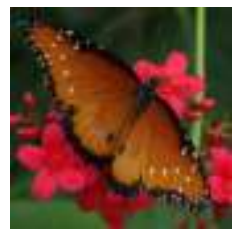

(d)

Figure 3: Cover images of size $256 \times 256$

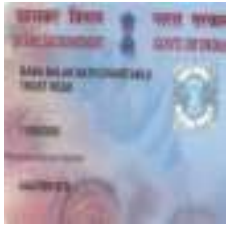

(a)

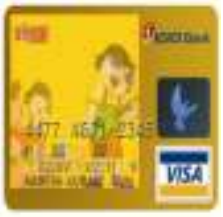

(b)
Figure 4: Secrete images of size $256 \times 256$

We have generated codebook of size $256 \times 12$ for cover image \& secret image. Then these two codebooks are combined giving $512 \times 12$ codebook using shuffle algorithm. There is only VQ distortion in reconstructing the image.

Table 1: Comparisons of the algorithms LBG, KPE KFCG with respect to MSE, PSNR for cover images

\begin{tabular}{|l|r|r|r|r|r|r|}
\hline & \multicolumn{2}{|c|}{ LBG } & \multicolumn{2}{c|}{ KPE } & \multicolumn{2}{c|}{ KFOG } \\
\hline & \multicolumn{1}{|c|}{ MSE } & PSNR & \multicolumn{1}{c|}{ MSE } & \multicolumn{1}{c|}{ PSNR } & MSE & PSNR \\
\hline Catdog & 164.79 & 25.96 & 160.39 & 26.08 & 128.72 & 27.03 \\
\hline Eleafrica & 148.53 & 26.41 & 142.40 & 26.60 & 111.18 & 27.67 \\
\hline Flower 1 & 123.44 & 27.22 & 114.32 & 27.55 & 94.39 & 28.38 \\
\hline Redbutterfly & 97.11 & 28.26 & 88.56 & 28.66 & 69.18 & 29.73 \\
\hline
\end{tabular}

Table 2: Comparisons of the algorithms LBG, KPE, KFCG with respect to MSE, PSNR for Secrete images

\begin{tabular}{|l|c|c|c|c|c|c|}
\hline & \multicolumn{2}{|c|}{ LBG } & \multicolumn{2}{c|}{ KPE } & \multicolumn{2}{c|}{ KFCG } \\
\hline & MSE & PSNR & MSE & PSNR & MSE & PSNR \\
\hline Pancad & 54.37 & 30.78 & 53.91 & 30.81 & 45.84 & 31.52 \\
\hline Greditcard & 151.09 & 26.34 & 150.42 & 26.36 & 119.53 & 27.36 \\
\hline
\end{tabular}

For the same cover images given in Figure 3 the following message by Abraham Lincoln is hidden. The Entire message is converted to codebook of size $256 \times 12$ and then proposed algorithm is used to hide the message codebook in the cover image codebook. To improve the secrecy of text message every byte of text message is ex-ored with key. The text message is extracted by ex-oring with same key.

\section{Hidden Message:}

Abraham Lincoln (February 12, 1809 - April 15, 1865) served as the 16th President of the United States from March 1861 until his assassination in April 1865. He successfully led his country through its greatest internal crisis, the American Civil War, preserving the Union and ending slavery. Before his election in 1860 as the first Republican president, Lincoln had been a country lawyer, an Illinois state legislator, a member of the United States House of Representatives, and twice an unsuccessful candidate for election to the U.S. Senate. He won an election to legislature. Find below a beautiful letter written by Abraham Lincoln.

Respected Teacher,

My son will have to learn I know that all men are not just, all men are not true. But teach him also that for ever scoundrel there is a hero; that for every selfish politician, there is a dedicated leader. Teach him that for every enemy there is a friend.

It will take time, I know; but teach him, if you can, that a dollar earned is far more valuable than five found.

Teach him to learn to lose and also to enjoy winning. Steer him away from envy, if you can.

Teach him the secret of quite laughter. Let him learn early that the bullies are the easiest to tick.

Teach him, if you can, the wonder of books. But also give him quiet time to ponder over the eternal mystery of birds in the sky, bees in the sun, and flowers on a green hill -side.

In school teach him it is far more honourable to fail than to cheat. Teach him to have faith in his own ideas, even if every one tells him they are wrong.

Teach him to be gentle with gentle people and tough with the tough.

Try to give my son the strength not to follow the crowd when every one is getting on the bandwagon.

Teach him to listen to all men but teach him also to filter all he hears on a screen of truth and take only the good that comes through.

Teach him, if you can how to laugh when he is sad. Teach him there is no shame in tears.

Teach him to scoff at cynics and to beware of too much sweetness. Teach him to sell his brawn and brain to the highest bidders; but never to put a price tag on his heart and soul. 
Teach him to close his ears to a howling mob... and to stand and fight if he thinks he's right.

Treat him gently; but do not cuddle him because only the test of fire makes fine steel. Let him have the courage to be impatient, let him have the patience to be brave.

Teach him always to have sublime faith in himself because then he will always have sublime faith in mankind. "This is a big order; but see what you can do. He is such a fine little fellow, my son".

- Abraham Lincoln

I wonder why teachers fail to implement this?

we often see teachers favouring bright students and the average students do feel

rejected and loose their confidence even if they have talent in them, the foundation of every student

is his primary education and I belive teachers teacher teaching in primary school should take these factors into consideration and develop each and every student and make a good citizen.

\section{CONCLUSION}

Existing algorithm gives limited hiding capacity since information is hidden inside index based cover image. But in our proposed approach hiding capacity is $100 \%$ or more since codebooks of cover image \& secret messages are combined using shuffle algorithm. No error is introduced except VQ distortion while reconstructing the image. If secret message is text, entire message is extracted without distortion. Secrecy of embedded message is improved since different VQ algorithms can be used for cover and secret image. As well as Conventional stegaanalysis techniques fail to detect secret message.

\section{REFERENCES}

[1] J. Fridrich, M. Goljan, and R. Du, "Lossless data embedding - new paradigm in digital watermarking," EURASIP J. Appl. Signal Processing, vol. 2002, no. 2, pp. 185-196, Feb. 2002.

[2] H. B. Kekre, Archana Athawale and Pallavi N.Halarnkar, "Increased Capacity of Information Hiding in LSBs Method for Text and Image", International Journal of Electrical, Computer and Systems Engineering, Volume 2 Number 4. http://www.waset.org/ijecse/v2.html.

[3] H. B. Kekre, Archana Athawale and Pallavi N.Halarnkar, "Polynomial Transformation To Improve Capacity Of Cover Image For Information Hiding In Multiple LSBs", International Journal of Engineering Research and Industrial Applications (IJERIA), Ascent Publications, Volume II, March 2009, Pune.

[4] H. B. Kekre, Archana Athawale and Pallavi N.Halarnkar, "Performance Evaluation Of Pixel Value Differencing And Kekre's Modified Algorithm For Information Hiding In Images", ACM International Conference on Advances in Computing, Communication and Control (ICAC3).2009 (Uploaded on ACM Portal: http://portal.acm.org/citation.cfm?id=1523103.1523172).

[5] S.D. Lin and C.F. Chen, A Robust DCT-based Watermarking for Copyright Protection, IEEE
Transactions on Consumer Electron, vol. 46, no. 3, pp. 415-421, 2000.

[6] Y.T. Wu and F.Y. Shih, Genetic algorithm based methodology for breaking the steganalytic systems, IEEE Transactions on Systems, Man and Cybernetics. Part B, vol. 36, no. 1, pp. 24-31, 2006.

[7] C. C. Chang, and C. Y. Lin, Reversible Steganography for VQ-compressed Images Using Side Matching and Relocation, IEEE Transactions on Information Forensics and Security, vol. 1, no. 4, pp. 493-501, 2006.

[8] C. C. Chang, Y. C. Chou and C. Y. Lin, Reversible Data Hiding in the VQ-Compressed Domain, IEICE Transactions on Information and Systems, vol. E90-D no. 9, pp. 1422-1429, 2007.

[9] Y. Linde, A. Buzo, and R. M. Gray, "An algorithm for vector quantizer design," IEEE Trans. Commun., vol. COM- 28, no. 1, pp. 84-95, 1980.

[10] A. Gersho, R.M. Gray.: 'Vector Quantization and Signal Compressio', Kluwer Academic Publishers, Boston, MA, 1991.

[11] H. B. Kekre, Tanuja K. Sarode, "New Fast Improved Codebook generation Algorithm for Color Images using Vector Quantization," International Journal of Engineering and Technology, vol.1, No.1, pp. 67-77, September 2008.

[12] H. B. Kekre, Tanuja K. Sarode, "An Efficient Fast Algorithm to Generate Codebook for Vector Quantization," First International Conference on Emerging Trends in Engineering and Technology, ICETET-2008, held at Raisoni College of Engineering, Nagpur, India, 1618 July 2008, Avaliable at online IEEE Xplore.

[13] H. B. Kekre, Tanuja K. Sarode, "Fast Codebook Generation Algorithm for Color Images using Vector Quantization," International Journal of Computer Science and Information Technology, Vol. 1, No. 1, pp: 7-12, Jan 2009.

[14] H. B. Kekre, Tanuja K. Sarode, "New Fast Improved Codebook Generation Algorithm for Color Images using Vector Quantization", International Journal of Engg. \& Tech., Vol.1, No.1, pp. 67-77, 2008.

[15] M. Jo and H. D. Kim, "A Digital Image Watermarking Scheme Based on Vector Quantisation," IEICE Trans. Inf. \& Syst., vol. E85-D, no. 6, June 2002, pp. 1054-1056.

[16] Chi-Nan Lin; Chin-Chen Chang;"A Best-pair-first Capacity-distortion Control for Data Hiding on VQ Compression Domain", Intelligent Information Hiding and Multimedia Signal Processing, 2007. IIHMSP 2007. Third International Conference on Volume 1, 26-28 Nov. 2007 Page(s):509 - 514

[17] A. Gersho and R. M. Gray, Vector Quantization and Signal Compression. Norwell, MA: Kluwer, 1992.

[18] Z. N. Li and M. S. Drew, Fundamentals of Multimedia. Englewood Cliffs, NJ: Prentice-Hall, Oct. 2003.

[19] N. M. Nasrabadi and R. King, "Image coding using vector quantization: A review," IEEE Trans. Commun., vol. 36, no. 8, pp. 957-971, Aug. 1988.

[20] C. H. LEE, L. H. CHEN, "Fast Codeword Search Algorithm for Vector Quantization", IEE Proceedings Image Signal Processing Vol 141, No. 3 June 1994. 
[21] H. B. Kekre, Tanuja K. Sarode, Bhakti Raul, "Color Image Segmentation using Kekre's Fast Codebook Generation Algorithm Based on Energy Ordering Concept", ACM International Conference on Advances in Computing, Communication and Control (ICAC3-2009), pp.: 357-362, 23-24 Jan 2009, Fr. Conceicao Rodrigous College of Engg., Mumbai. Available on ACM portal.

[22] H. B. Kekre, Tanuja K. Sarode, Bhakti Raul, "Color Image Segmentation using Kekre's Algorithm for Vector Quantization", International Journal of Computer Science (IJCS), Vol. 3, No. 4, pp.: 287-292, Fall 2008. Available: http://www.waset.org/ijcs.

[23] H. B. Kekre, Tanuja K. Sarode, Bhakti Raul, "Color Image Segmentation using Vector Quantization Techniques Based on Energy Ordering Concept" International Journal of Computing Science and Communication Technologies (IJCSCT) Volume 1, Issue 2, pp: 164-171, January 2009.

[24] H. B. Kekre, Tanuja K. Sarode, Bhakti Raul, "Color Image Segmentation Using Vector Quantization Techniques", Advances in Engineering Science Sect. C (3), pp.: 35-42, July-September 2008.

[25] H. B. Kekre, Tanuja K. Sarode, "Speech Data Compression using Vector Quantization”, WASET International Journal of Computer and Information Science and Engineering (IJCISE), vol. 2, No. 4, pp.: 251254, Fall 2008. available: http://www.waset.org/ijcise.

[26] H. B. Kekre, Ms. Tanuja K. Sarode, Sudeep D. Thepade, "Image Retrieval using Color-Texture Features from DCT on VQ Codevectors obtained by Kekre's Fast Codebook Generation", ICGST-International Journal on Graphics, Vision and Image Processing (GVIP), Volume 9, Issue 5, pp.: 1-8, September 2009 Available online at http://www.icgst.com/gvip/Volume9/Issue5/P1150921752. html.

[27] H. B. Kekre, Tanuja Sarode, Sudeep D. Thepade, "ColorTexture Feature based Image Retrieval using DCT applied on Kekre's Median Codebook", International Journal on Imaging (IJI), Volume 2, Number A09, Autumn 2009,pp. 55-65. Available online at www.ceser.res.in/iji.html (ISSN: 0974-0627).
[28] H. B. Kekre, Kamal Shah, Tanuja K. Sarode, Sudeep D. Thepade, "Performance Comparison of Vector Quantization Technique - KFCG with LBG, Existing Transforms and PCA for Face Recognition", International Journal of Information Retrieval (IJIR), Vol. 02, Issue 1, pp.: 64-71, 2009.

[29] H. B. Kekre, Tanuja K. Sarode, "2-level Vector Quantization Method for Codebook Design using Kekre's Median Codebook Generation Algorithm", Advances in Computational Sciences and Technology (ACST), ISSN 0973-6107, Volume 2 Number 2, 2009, pp. 167-178. Available online http://www.ripublication.com/Volume/acstv2n2.htm.

[30] H. B. Kekre, Tanuja K. Sarode, "Multilevel Vector Quantization Method for Codebook Generation", International Journal of Engineering Research and Industrial Applications (IJERIA), Volume 2, No. V, 2009, ISSN 0974-1518, pp.: 217-235. Available online at http://www.ascentjournals.com/ijeria_contents_Vol2No5.htm.

[31] H. B. Kekre, Tanuja K. Sarode "Vector Quantized Codebook Optimization using K-Means", International Journal on Computer Science and Engineering (IJCSE) Vol.1, No. 3, 2009, pp.: 283-290, Available online at: $\mathrm{http} / / /$ journals.indexcopernicus.com/abstracted.php?level= 4\&id_issue $=839392$.

[32] H. B. Kekre, Tanuja K. Sarode, "Bi-level Vector Quantization Method for Codebook Generation", Second International Conference on Emerging Trends in Engineering and Technlogy, at G. H. Raisoni College of Engineering, Nagpur on 16-18 December 2009, this paper will be uploaded online at IEEE Xplore.

[33] H. B. Kekre, Tanuja K. Sarode, "Bi-level Vector Quantization Method for Codebook Generation", Second International Conference on Emerging Trends in Engineering and Technlogy, at G. H. Raisoni College of Engineering, Nagpur on 16-18 December 2009, this paper will be uploaded online at IEEE Xplore. 